3. - Tests for the superadditivity of functions, Proc. Amer. Math. Soc. 13 (1962), 126-130.

4. A. Denjoy, Sur une propriete des fonctions dérivese, Enseignement Math. 18 (1916), 320-328.

5. E. Hille and R. Phillips, Functional analysis and semigroups, Chapter VII, pp. 237-255, Amer. Math. Soc. Colloq. Publ. Vol. 31, Amer. Math. Soc., Providence, R. I., 1957.

6. R. G. Laatsch, Subadditive functions of one real variable, Doctoral dissertation, Oklahoma State University, Stillwater, Oklahoma, 1962.

7. R. A. Rosenbaum, Subadditive functions, Duke Math. J. 17 (1950), 227-247.

University of California, Santa Barbara

\title{
ON ERGODIC MEASURES
}

YAEL NAIM DOWKER AND G. LEDERER

Introduction, notation, definitions and known results. In what follows $\Omega$ will be a compact metric space and $T$ a homeomorphism of $\Omega$ onto itself. The pair $(\Omega, T)$ is called a compact discrete dynamical system. If $m$ is a Borel measure in $\Omega$ such that $m(\Omega)=1, m$ is called a normalised Borel measure. Throughout this paper all measures will be assumed to be normalised Borel measures. If for any Borel set $B$, $m(B)=m(T B), m$ is called an invariant measure. Ergodic invariant measures will simply be referred to as ergodic measures. If, for some set $A, T(A)=A, A$ will be called an invariant set. A nonempty, closed, invariant set which has no proper subset of the same properties is called a minimal set.

The empty set will be denoted by $\varnothing$. Given two sets $A$ and $B$, ' $A \subseteq B$ ' will stand for ' $A$ is a subset of $B$ ', while ' $A \subset B$ ' for ' $A$ is a proper subset of $B$ '.

The set of points $p, T(p), T^{2}(p), \cdots$ is called the positive semiorbit of $p$ and will be denoted by $O_{p}^{+}$. The set of limit points of the sequence $p, T(p), T^{2}(p), \cdots$ in $\Omega$ is called the $\omega$-limit set of $p$ and will be denoted by $\omega_{p}$. The set of points $p, T(p), T^{-1}(p), T^{2}(p)$, $T^{-2}(p), \cdots$ is called the orbit of $p$ and will be denoted by $O_{p}$. The closure of the semiorbit (orbit) in $\Omega$ is called the semiorbit closure (orbit closure) and will be denoted by $\bar{O}_{p}^{+}\left(\bar{O}_{p}\right) . \mathfrak{N}(p, \rho)$ will denote the open sphere about $p$ of radius $\rho$.

Received by the editors October 18, 1962. 
$C(\Omega)$ will denote the space of all real-valued continuous functions defined on $\Omega$ with the norm $\|f(x)\|=\operatorname{Max}_{x \in \Omega}|f(x)|$.

For any $f(x)$ in $C(\Omega)$ put $F_{n}(x)=(1 / n) \sum_{i=0}^{n-1} f\left(T^{i}(x)\right)$. We call $p$ a mean point $(p \in Q)$, if $\lim _{n \rightarrow \infty} F_{n}(p)$ exists for every $f(x)$ in $C(\Omega)$. In that case we put $\lim _{n \rightarrow \infty} F_{n}(p)=F(p)$. The notion of a mean point is due to Krylov and Bogoljubov who called such a point a quasiregular point (cf. [2, p. 93]). They showed that $Q \neq \phi$, if $\Omega \neq \phi$. They also showed that to each mean point there corresponds a unique invariant measure. This is obtained in the following manner:

Fix a mean point $p$ and for any $f(x)$ in $C(\Omega)$ put $L(f)=F(p)$. Then $L(f)$ is a positive linear functional on $C(\Omega)$. Moreover, $L(f)$ is invariant, i.e., $L(f)=F(p)=F(T(p))=L(T f)$. Hence, by a well-known theorem of F. Riesz (cf. [4]), there is a unique measure $\mu$ in $\Omega$ such that $\mu$ is invariant and

$$
L(f)=\int_{\Omega} f(x) d \mu(x)
$$

for every $f(x)$ in $C(\Omega) . \mu$ is the invariant measure corresponding to $p$ which we shall denote by $\mu_{p}$.

A mean point $p$ is called transitive $\left(p \in Q_{T}\right)$ if $\mu_{p}$ is ergodic. A mean point $p$ is called dense $\left(p \in Q_{D}\right)$ if $\mu_{p}(U)>0$ for every open neighbourhood $U$ of $p$. We put $R=Q_{D} \cap Q_{T}$. Points of $R$ are called regular points.

We give now a few theorems due to Krylov and Bogoljubov. See [3] for a concise, comprehensive summary of these.

K.B. 1. $Q, Q_{T}, Q_{D}$ and $R$ are nonempty, invariant Borel sets, and for any invariant measure $m, m(R)=1$. Hence, there is at least one ergodic measure $\mu$ in $\Omega$. $\left(\mu=\mu_{p}\right.$ for some $p$ in $Q_{T}$.)

Not only does any point $p$ in $Q_{T}$ determine uniquely an ergodic measure $\mu_{p}$, but every ergodic measure $\mu$ in $\Omega$ is determined this way by some point $p$ of $R\left(R \subset Q_{T}\right)$. In this manner $Q_{T}$ can be decomposed into disjoint invariant sets, called quasi-ergodic sets, each of which consists of all transitive points which determine one and the same ergodic measure in $\Omega$. The intersection of each quasi-ergodic set with $R$ is nonempty, invariant and is called an ergodic set. Ergodic sets then form the corresponding decomposition of $R$. Thus

K.B. 2. There is a 1-1 correspondence between the class of ergodic measures in $\Omega$, the class of quasi-ergodic sets in $Q_{T}$ and the class of ergodic sets in $R$. In this correspondence, if $E$ is the ergodic and $E^{\prime}$ the quasi-ergodic set corresponding to the measure $\mu$, then $E$ and $E^{\prime}$ are Borel sets, $E \subseteq E^{\prime}, \mu=\mu_{p}$ for every $p$ in $E^{\prime}$, and $\mu(E)=1$. Moreover, for every Borel set $B, \mu_{p}(B)$ is a Borel-measurable function of $p$, and for any invariant measure $\mu$ 


$$
\mu(B)=\int_{R} \mu_{p}(B) d \mu(p)=\int_{Q_{T}} \mu_{p}(B) d \mu(p) .
$$

K.B. 3. If $A$ is a closed invariant set and $E$ any ergodic set, then either $A \cap E=E$ or $A \cap E=\varnothing$. In the former case $E$ is an ergodic set of $(A, T)$.

K.B. 4. If $E$ is an ergodic set and $p \in E$, then $E \subseteq \bar{O}_{p}$.

K.B. 5. If $E_{1}$ and $E_{2}$ are two distinct quasi-ergodic sets, there is a function $f(x)$ in $C(\Omega)$ and there are two distinct real numbers $c_{1}$ and $c_{2}$ such that $F(x)=c_{1}$ on $E_{1}$ and $F(x)=c_{2}$ on $E_{2}$.

The following theorem follows easily from the Krylov and Bogoljubov theory. See $[3$, p. 124] for a proof.

O. If all points of $\Omega$ are mean points and $M$ is a minimal set, then $M$ is an ergodic set.

The following theorems are due to one of us:

D. 1. If $p \in Q$, then $\mu_{p}\left(\bar{O}_{p}^{+}\right)=1$.

D. 2. If $p$ is not a transitive point, then $\bar{O}_{p}^{+}$contains at least two ergodic sets.

D. 3. Let $p \in \Omega$ and let $A$ be a nonempty, closed, invariant proper subset of $\bar{O}_{p}^{+}$. Let $U$ be an open set such that $A \subset U$ and $\bar{O}_{p}^{+} \Phi \bar{U}$. There is then a compact set $K$ such that $A \subset K \subseteq \bar{U}$ and $T K \subseteq K$.

For a proof of theorems D. 1, D. 2, and D. 3, see [1, pp. 126-128]. D. 1 and D. 2 are Lemmas 3 and 7, respectively. D. 3 is an immediate consequence of $\mathrm{K}$ and Lemma 8.

One of us has shown (cf. [1]) that if $\Omega$ has an infinity of points, it has an infinity of transitive points. In this paper we deal with the question of existence of an infinity of ergodic measures. It is knownas expressed in $\mathrm{O}$-that if $\Omega$ is itself a minimal set and all points in $\Omega$ are mean points, then there is only one invariant measure in $\Omega$. The question arises whether there can be more than one such measure in $\Omega$ if all points of $\Omega$ are mean points and $\Omega$ contains a single minimal set properly. While this problem is still unsolved, a partial answer is provided in this paper.

THEOREM. Let $(\Omega, T)$ be a discrete dynamical system. Let each point of $\Omega$ be a mean point and let $\Omega$ contain a single minimal set properly. Then the number of ergodic measures in $\Omega$ is either one or infinite.

Proof. Assume that the hypotheses of the theorem hold and denote by $M$ the minimal set. By K.B. 1 there is at least one ergodic measure in $\Omega$. Assume next that the number of ergodic measures in $\Omega$ is at least two. Then $\Omega$ has at least two ergodic sets of which $M$ is one (O). Let $k$ be the cardinal number of the class of ergodic sets of $\Omega$. 
Then $k \geqq 2$. Assume now that $k$ is finite. Denote by $E$ one of the ergodic sets of $\Omega(E \neq M)$. Fix a point $p$ in $E$. ( $p$ will denote the same point for the rest of the paper.) Then, by K.B. $4, E \subseteq \bar{O}_{p}$. Put $\bar{O}_{p}=X$. Then $X$ is an invariant, closed subset of $\Omega$. We confine our attention to the system $(X, T)$. The symbols $Q, R$ and $\mu_{q}$ (for any $q$ in $X)$ will refer to $X$. Clearly, all assumptions on $(\Omega, T)$ hold for $(X, T)$. By K.B. $3, X$ has at most $k$ ergodic sets. These clearly include $E$ and $M$. Denote the quasi-ergodic sets of $X$ by $E_{1}, E_{2}, \cdots$, $E_{j}\left(j \leqq k, E \subseteq E_{1}=E^{\prime}, M \subseteq E_{j}\right)$.

We assert now that $X=\omega_{p}$. To prove this, note that for any integer $n, T^{n}(p) \in E \subset R$, and hence if $U$ is an open neighbourhood of $T^{n}(p)$, $\mu_{p}(U)>0$. Now assume that $X \neq \omega_{p}$. There is then an open set $U$ contained in $X-\omega_{p}$ and there are integers $n_{0}$ and $N$ such that $T^{n_{0}}(p) \in U$ while $T^{n}(p) \in X-U$ for all $n \geqq N$. Hence, by D. $1, \mu_{p}(X-U)=1$, while $\mu_{p}(U)>0-$ which is absurd. Thus $X=\omega_{p}$ and hence $E$ is dense in $X$.

Now if $q \in E^{\prime}$, then $\mu_{q}=\mu_{p}$ in $X$. Hence if $q \in E^{\prime}$ and $U$ is any open neighbourhood of $q$, then since $T^{n}(p) \in U$ for some $n, \mu_{q}(U)=\mu_{p}(U)$ $>0$. Thus all points of $E^{\prime}$ are regular. Hence $E=E^{\prime}$.

We assert next that for $1<i \leqq j, E_{i}$ is nowhere dense in $X$. To prove this, assume that for some $i(1<i \leqq j) E_{i}$ is dense in an open sphere $U$ of $X$. Since $X=\omega_{p}$, there is an integer $n_{1}$ such that $T^{n_{1}}(p) \in U$. Now take any open sphere $V$ of $X$. Since $X=\bar{O}_{p_{n}}$, there is some integer $n_{2}$ for which $T^{n_{2}}(p) \in V$. Put $n=n_{2}-n_{1}$. Then $T^{n}\left(T^{1}(p)\right) \in V$. Hence $V \cap T^{n}(U)$ is a nonempty, open set. Since $T^{n}\left(E_{i}\right)=E_{i}$ and $E_{i}$ is dense in $U, E_{i}$ is dense in $T^{n}(U)$. Hence $E_{i} \cap V \cap T^{n}(U) \neq \varnothing$, i.e., $E_{i} \cap V \neq \varnothing$. Since $V$ was arbitrary, we conclude that if $E_{i}$ is not nowhere dense in $X$, it must be dense in $X$.

Now since $E_{1}$ and $E_{i}$ are distinct quasi-ergodic sets, by K.B. 5 there is a function $f(x)$, continuous on $X$, and there are real constants $c_{1}$ and $c_{i}$ such that $c_{1} \neq c_{i}, F(x)=c_{1}$ on $E_{1}$ and $F(x)=c_{i}$ on $E_{i}$. Now since all points of $X$ are mean points, $F(x)$ is defined for all $x$ in $X$. Then since $E_{1}$ is dense in $X$, if $E_{i}$ is also dense in $X, F(x)$ is discontinuous at every point of $X$. This is impossible, since $F(x)$ is of Baire class 1. Thus $E_{i}$ is not dense in $X$. It is therefore nowhere dense.

We conclude that the set $\bigcup_{i=2}^{j} E_{i}$ is nowhere dense.

Put $H=\bigcup_{i=2}^{j} E_{i}$. Then $\bar{H}$ is nowhere dense, closed and invariant. There is then a point $x$ in $X$ and a positive number $\rho$ such that $\mathfrak{N}(x, 2 \rho) \cap \bar{H}=\varnothing$. Put $U=X-[\Re(x, \rho)]-$. Clearly, $U$ is open. Moreover, since $X=\omega_{p}$, there is a positive integer $n$ for which $T^{n}(p)$ $\in \Re(x, \rho) \subseteq X-\bar{U}$. Thus 


$$
\bar{H} \subset U \quad \text { and } \quad \bar{O}_{p}^{+} \Phi \bar{U} .
$$

Then since $U$ is open and $\bar{H}$ is an invariant closed subset of $\bar{O}_{p}^{+}$, there is, by D. 3, a compact set $K$ such that

$$
\bar{H} \subset K \subseteq \bar{U} \text { and } T K \subseteq K .
$$

There is thus a point $q$ in $K-\bar{H}$, such that $\omega_{q} \subseteq \bar{O}_{a}^{+} \subseteq K \subset X$. Hence $\omega_{q} \neq X$. We have proved that if $x \in E^{\prime}$, then $\omega_{x}=X$. Hence $q \notin E^{\prime}=E$. Also $E$ is dense in $X, \omega_{q}$ is closed and $\omega_{q} \neq X$. Thus $E \Phi \omega_{q}$. Then, by K.B. 3, since $\omega_{q}$ is invariant and closed, $E \cap \omega_{q}=\varnothing$.

Since $q \notin E^{\prime}$ and $q \in K-\bar{H}, q \notin \cup_{i=1}^{\prime} E_{i}$. Hence $q$ is not transitive nor is any point of $O_{q}$. Thus, by D. $2, \bar{O}_{q}^{+}$contains at least two ergodic sets. Clearly, $M$ is one of these. Also, no point of $O_{q}$ is regular. Thus $\omega_{q}$ contains at least two ergodic sets of which $M$ is one, while $E \cap \omega_{q}$ $=\varnothing$. Put $\omega_{q}=\Omega_{1}$. Then $\Omega_{1}$ is an invariant, closed, proper subset of $\Omega$, consisting of mean points only, containing a single minimal set properly and containing (by the above and by K.B. 3) at least two and at most $k-1$ ergodic sets.

The process can be repeated any number of times yielding a decreasing sequence $\Omega, \Omega_{1}, \Omega_{2}, \cdots, \Omega_{r}, \cdots$ of invariant closed sets, where for each $r \Omega_{r}$ contains at least two and at most $k-r$ ergodic sets. A contradiction is obtained for $r=k-1$. Our assumption that $k$ is finite was false and the theorem is proved.

COROLLARY. Let the hypotheses of the theorem hold. Then, if there are two distinct invariant measures in $\Omega$, there is an infinity of distinct ergodic measures in $\Omega$.

Proof. By K.B. 2, if there is only one ergodic measure in $\Omega$, there can be only one measure in $\Omega$. The corollary follows now from the theorem.

\section{REFERENCES}

1. Y. N. Dowker, The mean and transitive points of homeomorphisms, Ann. of Math. (2) 58 (1953), 123-133.

2. $\mathrm{N}$. Krylov, and N. Bogoljubov, La théorie générale de la mesure dans son application à l'étude des systèmes de la mécanique non linéaire, Ann. of Math. (2) 38 (1937), 65-113.

3. J. C. Oxtoby, Ergodic sets, Bull. Amer. Math. Soc. 58 (1952), 116-136.

4. S. Saks, Integration in abstract metric spaces, Duke Math. J. 4 (1938), 408-411.

The University, ShefField AND

IMPERIAL COLLEGE 